\title{
Deep brain stimulation induced effects in a network of ventral intermediate neurons
}

\author{
Caroline T Golden ${ }^{1}$, Dipankar Nandi', Peter Bain ${ }^{2}$, Nada Yousif ${ }^{*}$ \\ From Twenty Second Annual Computational Neuroscience Meeting: CNS*2013 \\ Paris, France. 13-18 July 2013
}

Essential tremor (ET) is a common movement disorder successfully treated by deep brain stimulation (DBS) [1], a surgical therapy involving the implantation of electrodes into disorder specific nuclei in the brain through which high frequency electrical pulses are chronically administered. While the therapy is clinically beneficial, the mechanisms at the level of the changes occurring in the surrounding tissue are unknown [2]. If we better understood these mechanisms, we could optimise the parameters setting process and minimise unwanted side effects in patients. To investigate these mechanisms we model a small network of excitatory relay cells and inhibitory interneurons within the ventral intermediate (Vim) nucleus of the thalamus. The network model was constructed using previously published multicompartment models [3] in NEURON 7.0 and was set up to reflect the known connectivity of the Vim nucleus. The DBS induced electric field was simulated in a finite element environment using COMSOL Multyphysics 3.3 by solving the Laplace equation within a 3-dimensional geometric model of the DBS electrode within a homogenous cylinder of brain tissue. The findings show that a variety of neural activity patterns are induced by therapeutic and non-therapeutic parameters derived from empirical and clinical reports. The network in isolation displayed tonic firing patterns in most of the relay cells, with strong inhibition in the remaining excitatory cells. The prevalence of interneuron-relay synaptic connections may be influential in this respect by acting as a gain control mechanism for relay cells, a concept which has been alluded to in prior investigations [4]. Once DBS was applied (Figure 1A), the network behaviour changed considerably. A combined effect of excitation and inhibition, which was dependent on spatial positioning within the stimulus field, decreased the overall activity of the network and increased the prevalence of hyperpolarisation-induced burst firing at DBS offset (Figure 1A). The simulations revealed this to be one of the most discernible network-level changes between therapeutic and non-therapeutic DBS simulations, which may relate to the links made in the literature between burst firing and tremor oscillations [5]. This is the first time that the effect of the DBS induced electric field on a thalamic network has been investigated and leads to a clearer understanding of the neuronal changes in the VIM nucleus.

\section{Acknowledgements}

NY is funded by a medical research grant from the Bupa foundation.

\section{Author details}

${ }^{1}$ Department of Bioengineering, Imperial College London, London, SW7 2AZ, UK. ${ }^{2}$ Division of brain sciences, Imperial College London, London, W86 8RF, UK.

Published: 8 July 2013

\section{References}

1. Benabid AL, Benazzouz A, Hoffmann D, Limousin P, Krack P, Pollak P: Longterm electrical inhibition of deep brain targets in movement disorders. Mov Disord 1998, 13:119-125.

2. Kringelbach ML, Jenkinson N, Owen SL, Aziz TZ: Translational principles of deep brain stimulation. Nat Rev Neurosci 2007, 8(8):623-635.

3. Destexhe A, Neubig M, Ulrich D, Huguenard J: Dendritic low-threshold calcium currents in thalamic relay cells. J Neurosci 1998, 18(10):3574-3588.

4. Sherman SM, Guillery RW: Functional organization of thalamocortical relays. J neurophysiol 1996, 76(3):1367-1395.

5. Lozano AM: Vim thalamic stimulation for tremor. Arch Med Res 2000, 31(3):266-269.

doi:10.1186/1471-2202-14-S1-P427

Cite this article as: Golden et al:: Deep brain stimulation induced effects in a network of ventral intermediate neurons. BMC Neuroscience 2013 14(Suppl 1):P427.

* Correspondence: n.yousif@imperial.ac.uk

${ }^{2}$ Division of brain sciences, Imperial College London, London, W86 8RF, UK

Full list of author information is available at the end of the article

(c) 2013 Golden et al; licensee BioMed Central Ltd. This is an Open Access article distributed under the terms of the Creative Commons Attribution License (http://creativecommons.org/licenses/by/2.0), which permits unrestricted use, distribution, and reproduction in any medium, provided the original work is properly cited. 


\section{Network Raster plot}

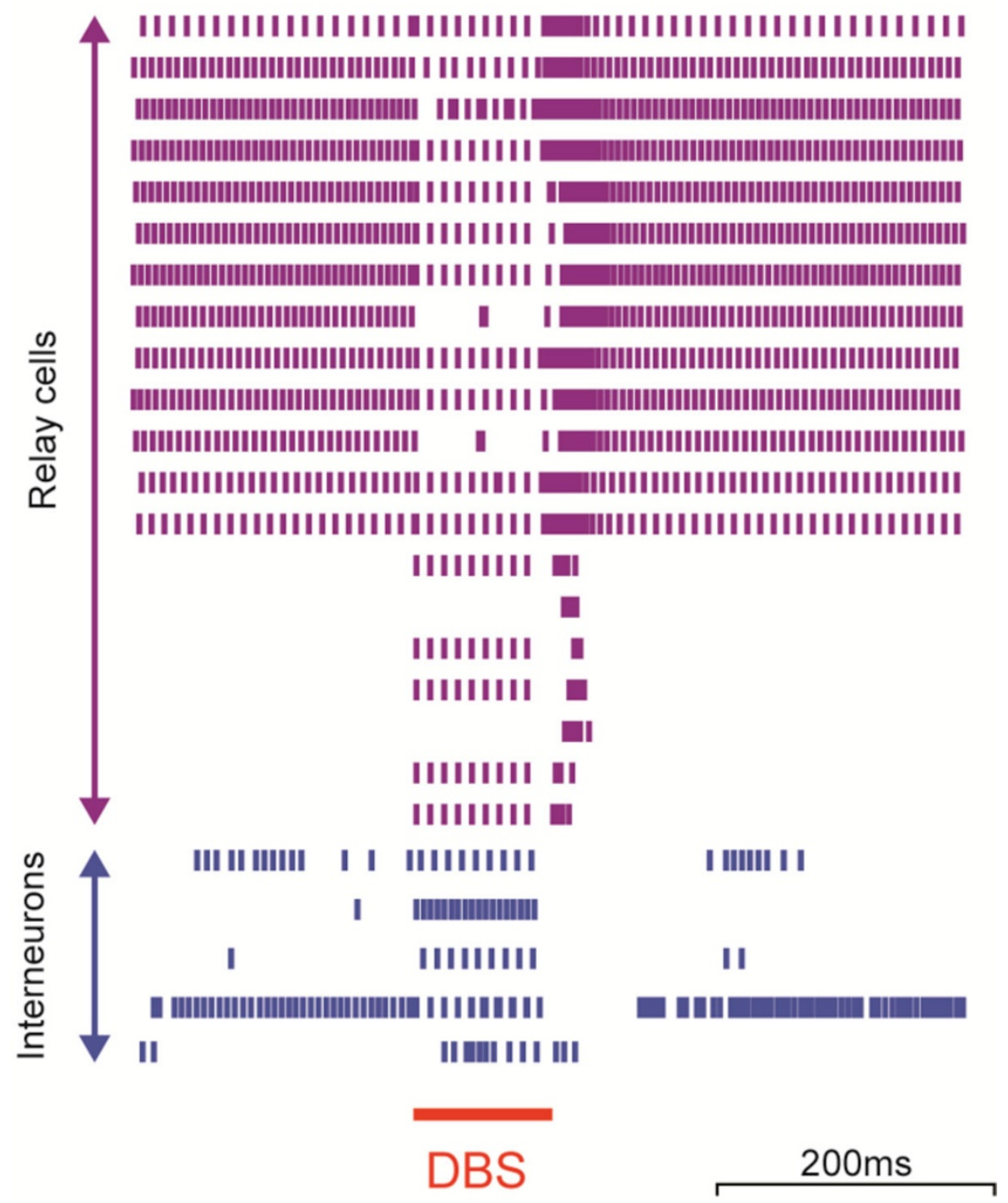

Figure 1 Raster plot of the modelled Vim nucleus network behaviour for a therapeutic DBS parameter setting (frequency $=100 \mathrm{~Hz}$, pulse width $=60 \mu \mathrm{s}$ and amplitude $=\mathbf{2 V}$ ). The 20 relay cells are shown with the five interneurons. The red line indicates the time during which DBS was simulated as being on (100 ms). 